Bamber, A; Pryce, J; Mifsud, W; Wolfe, I; Cass, H; Malone, M; Sebire, NJ; (2015) Potentially preventable infant and child deaths identified at autopsy; findings and implications. Forensic Science Medicine and Pathology 10.1007/s12024-015-9681-9.

\title{
Article
}

\section{Potentially Preventable Infant and Child Deaths Identified at Autopsy; Findings and Implications}

\author{
Andrew R. Bamber ${ }^{(1)}$,William Mifsud ${ }^{(1)}$,Ingrid Wolfe ${ }^{(2)}$,Hilary Cass ${ }^{(2)}$,Jeremy Pryce ${ }^{(1)}$ \\ ,Marian Malone ${ }^{(1)}$, Neil J. Sebire $\square^{(1)}{ }^{(3)}$. \\ 1. Department of Histopathology, Camelia Botnar Laboratories, Great Ormond Street \\ Hospital for Children NHS Foundation Trust, Great Ormond Street, London, WC1N 3JH, UK \\ 2. Evelina London Children's Hospital, Guy's and St Thomas' NHS Trust, Westminster \\ Bridge Road, London, SE1 7EH, UK \\ 3. Department of Paediatric Pathology, Great Ormond Street Hospital, London, WC1N 3JH, \\ UK
}

Key words: Autopsy, preventable, death, infant, child

\begin{abstract}
Purpose: To determine the proportion of pediatric deaths investigated by HM Coronial autopsy, which were potentially preventable deaths due to treatable natural disease, and what implications such findings may have for health policy to reduce such deaths.
\end{abstract}

Methods: Retrospective Study of 1,779 autopsies of individuals between 7 days and 14 years of age requested by HM Coroner, taking place in one specialist pediatric autopsy centre. Cases were included if they involved a definite natural disease process, in which appropriate recognition and treatment was likely to have affected outcome. Strict criteria were used and cases were excluded where the individual had any longstanding condition which might have predisposed to, or altered the recognition of, acute illness, or its response to therapy.

Results: Almost $8 \%(134 / 1779)$ of this population were potentially preventable deaths as a result of natural disease, the majority below the age of two years. Most individuals reported one to seven days of symptoms before death and the majority had sought medical advice during this period, including from general practitioners within working hours, and hospital emergency departments. Of those who had sought medical attention, around one third had more than once $(28 \%, 15 / 53)$. Sepsis and pneumonia accounted for the majority of deaths (46\% and $34 \%$ respectively), with all infections (sepsis, pneumonia and meningitis) accounting for $110 / 134(82 \%)$.

Conclusion: Around $10 \%$ of pediatric deaths referred to HM Coroner are potentially preventable, being the result of treatable natural acute illnesses. In many cases medical advice had been sought during the final illness. The results highlight how autopsy data review can identify findings of potential significance to mortality reduction, and the importance of centralised investigation and reporting of pediatric deaths. 


\section{Introduction}

The United Kingdom has the highest all cause mortality for children aged 0-14 in Western Europe[1]. Compared to Sweden, the best performing country, this is an annual excess of almost 2000 deaths, representing more than 132,000 Potential Years of Life Lost . The National Health Service (NHS) Outcomes Framework highlights areas for improvement including domain One ('Preventing people from dying prematurely') including 'Potential Years of Life Lost from causes considered amenable to healthcare in children and young people' (1aii).[2,3], and time from first NHS presentation to diagnosis or start of treatment has been suggested as an indicator of health care quality[4].

Case analysis of childhood deaths offers some insight into whether deaths were potentially avoidable. For example, the most recent Department of Education Child Death review report reported that $22 \%$ of deaths were identified as having potentially modifiable factors.[5] In addition, apparent 'missed diagnoses' have been repeatedly reported in the press, with particular attention on 'out of hours' primary care providers [6-8].

The aim of this study was to use post-mortem data to investigate infant and childhood deaths who were referred for autopsy at a large regional pediatric pathology centre, with a view to identifying those with a potentially preventable or treatable, natural cause of death in relation to preceding medical intervention. The study also aimed to explore the utility of using high quality autopsy data as a means of improving understanding of the findings of population based data, in order to potentially aid informing policy and ultimately to reduce avoidable mortality in children.

\section{Methods}

Great Ormond Street Hospital, London, is a specialist pediatric hospital which carries out autopsies on behalf of $\mathrm{HM}$ Coroners in the South of England together covering approximately 18 million of the population (25-30\% of total population)[9]. In the region, cases referred to HM Coroner include all sudden and/or unexpected deaths, and in most cases an autopsy will be requested. (Whilst a substantial proportion of autopsies in this area are referred to the centre, cases may also be sent to other centres for autopsy hence the dataset does not include $100 \%$ ascertainment of deaths in the region).

An autopsy database containing detailed non-identifiable data from every autopsy performed at the centre between January 1996 and January 2012 was searched to identify cases in which the cause of death was deemed to be a natural disease process in which preceding medical intervention was likely to have affected the outcome, including clear acute conditions such as pneumonia, appendicitis, volvulus, intussusception, meningitis and sepsis, following coroners' autopsies in infants and children aged 7 days to 14 years of age.

Strict inclusion and exclusion criteria were applied in order to ensure as far as possible that those included were otherwise normal infants and children dying of acute medical causes potentially amenable to intervention (Box 1). No cases were included in whom there was an underlying condition which might have either predisposed to disease, or changed the timecourse of disease, the recognition of severe illness or the response to therapy, and cases previously admitted to hospital during the index illness were also excluded. The data therefore underestimate the true frequency of all potentially preventable deaths in the group. Characteristics including symptoms, underlying diagnosis, medical advice sought, and treatment given were analysed for general trends rather than individual examples of possible suboptimal care. The study using routine non-identifiable autopsy data was approved by the local Research Ethics Committee (Bloomsbury REC). 


\section{Results}

Of 1,779 Coronial autopsies performed at the centre between 1996 and end 2011, $134(8 \%)$ fulfilled the strict inclusion criteria noted above. Nearly two-thirds were infants $(85 / 134,63 \%)$, and three-quarters were under the age of two years (100/134, 74\%; Figure 1) Causes of death are provided in Figure 2 . Sepsis and pneumonia accounted for the majority $(46 \%$ and $34 \%$ respectively), with all infections (sepsis, pneumonia and meningitis) accounting for $110 / 134(82 \%)$. A presumed pathogenic organism was isolated from peri-mortem or autopsy samples in $82 \%$ (Figure 3), the most common being Staphylococcus aureus $(21 / 134,15 \%)$, Group B Streptococcus (17/134, 13\%), Neisseria meningitidis (15/134, 11\%), Streptococcus pyogenes (Group A Streptococcus, 11/134, 8\%), and Streptococcus pneumoniae (9/134, $7 \%)$. Multiple organisms were implicated in 11 cases $(8 \%)$. Seven cases involved viral infection with bacterial super-infection (Table 1).

The duration of symptoms, as provided from the history and associated clinical notes, is provided in Figure 4. The majority described symptoms for between one and seven days (89/134, 66\%); there was a peak of individuals with duration of symptoms given as seven days, probably due to rounding duration of symptoms to a period of one week. The duration was either not provided or unclear in $16(12 \%)$. In 21 , there were apparently no symptoms preceding the final presentation (16\%), the majority of these cases involving sepsis (14/21, $58 \%)$, the others including pneumonia $(9 / 21,38 \%)$, and volvulus $(1 / 21,4 \%)$.

Patients were considered to have sought medical advice or treatment if they were seen and discharged before death, (the presentation or admission during which death occurred was not included); definite information regarding care prior to death was provided in 86 cases. In 33 of these it was recorded that no medical advice had been sought during the last illness. Advice was sought at least once from a medical professional or a professional allied to medicine in $62 \%$ (53/86; together totalling 71 consultations). General practitioners and hospital emergency departments accounted for $87 \%(62 / 71)$ of medical contacts during the final illness, whilst 'out of hours' general practitioners had been consulted only four times (Figure 5). Of those who sought medical attention, almost one third (15/53, 28\%) had sought medical advice on two or more occasions. One had sought medical attention on four occasions during the last illness prior to death (Figure 6). The number of attendances with medical professionals by cause of death is given in Figure 7. In nine of twelve cases of meningitis for which the number of attendances is recorded, the family had sought medical attention at least once during the last illness (75\%), one had sought advice twice, and one had sought advice three times without the condition being recognised. Treatment given prior to final admission, whether on the advice of medical professionals or empirically by the carer, is given in Figure 8.

\section{Discussion}

At present, autopsy derived data represents the gold standard for determining cause of death, since death certification alone is associated with a significant error rate (at least $30 \%$ for major errors)[10]. This study demonstrates how collated high-quality autopsy data can be a useful addition to case note analyses of health care practices in child death review processes, and may be useful for shaping policy responses, such as provision of paediatric services. At present, however, there is no standardised system for reporting and collating such autopsy findings from multiple centres. Large national databases allowing complete ascertainment would provide useful public health data.

The findings of this study demonstrate several important points. First, almost $10 \%$ of deaths in infants and children investigated by HM Coroner are potentially preventable, being the result of of treatable natural diseases. These most commonly affect children less than two 
years of age. It is widely accepted that the youngest children are often the most challenging for promptly detecting serious illness. Secondly, in most cases there were between one and seven days of symptoms preceding death, and in many cases there were multiple presentations to general practitioners and hospital emergency departments, such attendances representing potential opportunities for specialist referral or treatment. Thirdly, such contacts in primary care are not limited to 'out of hours' providers, but predominantly involve 'in hours' general practitioners and hospital emergency departments. Finally, some specific entities, such as life-threatening Group A Streptococcal Septicaemia arising in Varicella infection, highlight the importance of questioning primary diagnoses, and of providing adequate procedures to identify children who subsequently deteriorate.

These data demonstrate that over the period of the study, at least 134 infants and young children referred to our centre for autopsy were previously healthy and died of diseases which may have responded to appropriate medical treatment. Assuming that the relative frequency of these findings is similar to national data, there could be around 500 child deaths in the UK during the study period from infectious and gastrointestinal diseases which are potentially preventable. Indeed, since Coroners also send some cases to other units, and we have used extremely strict inclusion and exclusion criteria with the intention of highlighting deaths only in clearly previously healthy infants and children, our data purposely represent a significant under-estimate. We have not included any individuals born prematurely, or those with chronic medical conditions, and there will certainly also be potentially preventable deaths in these groups. Indeed, pre-existing medical conditions may make detection of superimposed significant serious illness even more difficult than in healthy children due to background symptoms. Furthermore, we have specifically excluded natural deaths which are the result of pre-existing medical conditions, which are nonetheless, potentially preventable, such as deaths due to asthma, a major cause of potentially preventable pediatric death.

The estimates arising from this study vary slightly from rates of preventable deaths given by previous reports. Data from the Child Death Overview Panels[11] suggests that preventable deaths due to natural diseases represent $2-3 \%$ of pediatric deaths. This is significantly less than the estimate arising from this study, most likely because CDOP data also includes 'expected' deaths which are not referred for autopsy, of which a smaller proportion are acute and potentially preventable. The report of CEMACH suggests as many as $26 \%$ of infant and childhood deaths result from a failure of care[5]. This is likely to be greater than the findings of the present study due to the inclusion of individuals with chronic diseases and conditions which might make the identification and treatment of severe illness more difficult.

Our results demonstrate that potentially preventable natural deaths are most common in younger children, with three-quarters affecting children under the age of two years. This may be a consequence of both innate predisposition to infectious diseases in this group and the fact that this is the age group who have the most difficulty communicating their symptoms. Furthermore signs can be subtle in infants and young children, and the natural course of illnesses in the young may include a rapid onset and precipitous decline. The data on duration of symptoms in the period preceding death, as given in the information provided at the time of autopsy, shows that a minority report no symptoms prior to sudden unexpected death; most of these cases representing systemic infection (sepsis) in young children and infants. However, it should be noted that a significant number of these cases involved a diagnosis of histologically-confirmed pneumonia and it seems unlikely that such individuals would have no symptoms at all; these cases may therefore result from a lack of recognition of the severity of illness on the part of some carers. Improved education regarding recognising the signs and symptoms of serious illness in young children may have an important contribution to reducing such deaths in addition to addressing issues of health service provision. 
Nevertheless, the majority of cases were associated with recognised preceding symptoms of illness, with carers of two-thirds of cases reporting symptoms of between one and seven days duration, giving a potential period for recognition and treatment. Indeed, of those where specific data was provided, the majority had sought medical advice or treatment, and of these, around one third had sought medical advice on at least two occasions prior to the presentation resulting in death. Of particular note, $75 \%$ of cases with autopsy-proven bacterial meningitis in which medical attendance had been recorded, had been seen by a medical professional at least once during their final illness. A previous study reporting on $>300$ cases of meningococcal disease reported that only half of those affected were referred to hospital following their initial presentation to medical care[12]. The data also demonstrate that, in contrast to portrayal in popular media, such missed opportunities are not usually a feature of 'out of hours' primary care providers; the vast majority of medical attendances were to hospital emergency departments or to general practices during normal working hours.

Finally, it is of note that seven children died of bacterial super-infection associated with previous viral infection, and notably, three of these cases involved Group A streptococcal septicaemia in the context of Varicella infection. This is a well-recognised complication of Varicella[13], and suspicion should be raised in cases of children attending primary care who are disproportionately unwell with otherwise typical signs of Varicella infection. Such examples represent potentially missed opportunities to treat a rare but well-reported lifethreatening condition.

The intention of the present study was not to assess individual practice, but rather to use high quality autopsy data to identify common risk factors at system level. We acknowledge that there are limitations, in particular, in the absence of a full investigation into the circumstances of death and the final illness, some of the cases we have selected as 'potentially preventable' may not have actually been prevented despite any form of medical care. However, since there is currently no method by which data such as this is routinely collated, our autopsy experience provides some data regarding this issue to raise awareness and stimulate debate. The data supports the suggestion that routinely including information such as the number of attendances to health care providers, past medical history, therapy given and specific causes of death, would allow similar analyses to be carried out nationally. The study also highlights the role of high quality autopsy data to provide important public health issues. Provision of accurate denominator data would allow more accurate estimates to be determined; at present it is not possible to derive exactly what proportion of infant and child deaths and referred to, or accepted by, HM Coroners, and what proportion of these cases have a potentially preventable cause identified at autopsy. Overall, around $45 \%$ of all UK deaths are referred to HM Coroner, and about $40 \%$ of these undergo autopsy examination, but no such specific data are available by age group, although children $<14$ years of age represents around $4 \%$ of inquests, (whilst representing $18 \%$ of the population)[14]. In addition, identification of cause of death in infant and child deaths is related to the expertise of the pathologist performing the examination, with more medical causes identified by specialist pathologists, hence further complicating registry derived data interpretation [15].

In summary, this autopsy study has demonstrated that around $8 \%$ of this population were potentially preventable deaths as a result of treatable natural disease, the majority affecting children below the age of two years. Most individuals reported one to seven days of symptoms before death and the majority had sought medical advice during this period, including from general practitioners within working hours, and hospital emergency departments. Such attendances represent potential missed opportunities for disease recognition and treatment. Sepsis and pneumonia accounted for the majority of deaths 


\section{References}

1. Wolfe I, Thompson M, Gill P, Tamburlini G, Blair M, van den Bruel A, et al. Health services for children in western Europe. Lancet. 2013;381:1224-34.

2. Department of Health. The NHS Outcomes Framework 2013/14. Available from:https://www.gov.uk/government/uploads/system/uploads/attachment_data/file/127106/1 21109-NHS-Outcomes-Framework-2013-14.pdf.pdf.

3. Department of Health. Improving outcomes and supporting transparency Part 2: Summary Technical Specifications of public health indicators. Available from:https://www.gov.uk/government/uploads/system/uploads/attachment_data/file/127179/I mproving-outcomes-and-supporting-transparency-part-21.pdf.pdf.

4. Children and Young People's Health Outcomes Strategy: Report of the Children and Young People's Health Outcomes forum. Available from:https://www.gov.uk/government/uploads/system/uploads/attachment_data/file/156062/ CYP-report.pdf.pdf.

5. https://www.gov.uk/government/uploads/system/uploads/attachment_data/file/332619/SFR2 1_2014_revised.pdf.

6. Lawrence $F$ and agencies. Mother tells inquest how baby died in care of privatised GP service. Guardian.co.uk 25 Feb 2013. Available from: http://www.guardian.co.uk/society/2013/feb/25/mother-court-privatised-gp-service.

7. Smith L. Sent home THREE times to die: The little boy who was a tragic victim of dehydration. Mirror.co.uk. 16 Apr 2012. Available from: http://www.mirror.co.uk/news/reallife-stories/little-harry-connolly-was-sent-home-796048.

8. Gornall J. Dozens like Kelsey have died through incompetent out-of-hours cover for their GPs. So how safe are YOU? Dailymail.co.uk. 10 Jul 2012. Available from:http://www.dailymail.co.uk/health/article-2171173/Dozens-like-Kelsey-diedincompetent-hours-cover-GPs-So-safe-YOU.html.

9. Office for National Statistics. 2011 Census: Usual Resident Population and population density, local authorities in the United Kingdom, Table 2. Available from: http://www.ons.gov.uk/ons/rel/census/2011-census/population-and-household-estimates-forthe-united-kingdom/rft-table-2-census-2011.xls.

10. Roulson J, Benbow EW, Hasleton PS. Discrepancies between clinical and autopsy diagnosis and the value of post mortem histology; a meta-analysis and review. Histopathology. 2005;47:551-559.

11. Department for Education Statistical Release: PREVENTABLE CHILD DEATHS IN ENGLAND: YEAR ENDING $31 \mathrm{MARCH} 2010$. Available from: https://www.gov.uk/government/publications/preventable-child-deaths-in-england-yearending-31-march-20107. 15

12. Thompson MJ, Ninis N, Perera R, Mayon-White R, Phillips C, Bailey L, et al. Clinical recognition of meningococcal disease in children and adolescents. Lancet. 2006;367:397403.

13. Cowan MR, Primm PA, Scott SM, Abramo TJ, Wiebe RA. Serious group A beta-hemolytic streptococcal infections complicating varicella. Ann Emerg Med 1994;23:818-22.

14. Ministry of Justice Statistical Bulletin and Tables. Coroners Statistics 2011 England and Wales. Available from: https://www.gov.uk/government/publications/coroners-statistics-ns.

15. Fleming PJ, Blair PSP, Bacon C, Berry PJ. 2000, Sudden Unexpected Death in Infancy. The CESDI SUDI Studies 1993-1996. The Stationary Office, London. 


\section{Figure legends}

Box 1: Inclusion and Exclusion Criteria

*For a diagnosis of sepsis any two of the following were required: Positive blood/spleen cultures, clinical evidence of sepsis, pathological evidence of sepsis.

**Allow if single previous infection, completely resolved before the onset of these symptoms.

${ }^{* * *}$ Allow if Mild neonatal problems, completely resolved (e.g. neonatal jaundice requiring phototherapy).

Figure 1.

Age distribution of 134 infants and children with potentially preventable deaths due to treatable natural causes and undergoing autopsy between January 1996 and December 2011

\section{Figure 2.}

Relative proportions of different causes of death in 134 infants and children with potentially preventable deaths due to treatable natural causes and undergoing autopsy between January 1996 and December 2011

\section{Figure 3.}

Primary Causative Organism in infants and children with potentially preventable deaths due to treatable natural causes and undergoing autopsy between January 1996 and December 2011

\section{Figure 4.}

Duration of symptoms (in days) as reported in the documentation provided at the time of autopsy in 134 infants and children with potentially preventable deaths due to treatable natural causes and undergoing autopsy between January 1996 and December 2011

\section{Figure 5.}

Medical professionals consulted during the final illness by 53 infants and children with potentially preventable deaths due to treatable natural causes and undergoing autopsy between January 1996 and December 2011

* Includes one patient who was refused an appointment and given advice by a GP receptionist over the telephone

$\S$ Includes one patient who telephoned the $\mathrm{OOH}$ GP and was refused an appointment a Clinical history states patient sought medical attention, but does not specify from whom

\section{Figure 6.}

Number of attendances with medical professionals during the last illness of 53 infants and children with potentially preventable deaths due to treatable natural causes and undergoing autopsy between January 1996 and December 2011

\section{Figure 7.}

Number of attendances with medical professionals by cause of death in134 infants and children with potentially preventable deaths due to treatable natural causes and undergoing autopsy between January 1996 and December 2011

\section{Figure 8.}

Therapy given to 134 infants and children with potentially preventable deaths due to treatable natural causes and undergoing autopsy between January 1996 and December 2011. [Two patients received symptom control and antibiotics] 